\title{
Thermodynamical Study of Alcoholic Solutions of Poly (ethylene glycol) Diacrylate and Poly (ethylene glycol) Dimethacrylate
}

\author{
Maimoona Yasmin* and Manisha Gupta \\ Department of Physics, University of Lucknow, Lucknow, India- 226007 \\ e-mail; myasmin908@gmail.com
}

\begin{abstract}
With the aim to study the behaviour of polymer solutions, density and ultrasonic velocity of binary mixtures of pentanol with polyethylene glycol diacrylate and polyethylene glycol dimethacrylate have been investigated at 293.15 K, 303.15 K and 313.15 K, under atmospheric pressure. Thermophysical parameters viz. intermolecular free length, internal pressure and pseudo-Grüneisen parameter have been calculated which infer about the interactions and structural changes on mixing pentanol with these polymers. Enthalpy, entropy and Gibbs free energy of activation for viscous flow at different concentrations of polymer solution have been reported. Excess molar volume, deviation in isentropic compressibility, deviation in ultrasonic velocity, excess acoustic impedance and excess molar enthalpy have been calculated and results have been correlated with Redlich-Kister polynomial equation. Density and ultrasonic velocity data have been fitted by third order polynomial equation.
\end{abstract}

\section{Keywords: Poly (ethylene glycol) diacrylate; poly (ethylene glycol) dimethacrylate; density; ultrasonic velocity.}

\section{Introduction}

Polyethylene glycol diacrylate (PEGDA) is a low volatility and medium viscosity clear liquid with good flexibility and elongation, good water dispersible property, low skin irritancy and good reactivity. It is used in flexible coatings, overprinting varnishes, offset inks and photopolymeric printing plates. PEGDA is soluble in water and is used as a functional co-monomer for flexible plastics and as a crosslinking agent between the molecular chains of polymers. PEGDA is able to effectively seal the vessel puncture sites and anastomotic junctions without acutely augmenting local vascular thrombogenicity (G.A. Dumanian et al.,1995).

Polyethylene (glycol) dimethacrylate (PEGDMA) is used in adhesives, coatings, sealants, photoresists, solder masks and photopolymers. It also contributes to flexibility.

1-Pentanol is a colorless liquid. To reduce the use of fossil fuels, research is underway to discover cost effective methods of utilizing fermentation to produce Bio-Pentanol. Pentanol can be used as a solvent for coating CDs and DVDs. Another use is a replacement for gasoline.

Such importance of these polymers and their solutions and its vast domain of industrial and biochemical applications have enforced us to study the means of predicting their properties. In the present work solutions of PEGDA and PEGDMA in pentanol have been studied. The aim of this work is to obtain information about the association among the components of mixtures under study, to investigate correlations among them and to provide qualitative interpretation in terms of molecular interactions. To our knowledge no density and velocity data have previously been reported for these mixtures.

Densities and ultrasonic velocities for the mixtures of pentanol + PEGDA and pentanol + PEGDMA have been reported at $293.15 \mathrm{~K}, 303.15 \mathrm{~K}$ and $313.15 \mathrm{~K}$, under atmospheric pressure. Derived parameters such as intermolecular free length, internal pressure, pseudo-
Grüneisen parameter, enthalpy, entropy and Gibbs free energy of activation for viscous flow at different concentrations of polymer solution have also been calculated using experimental data for both the systems. The intermolecular interactions present in the mixtures have been investigated through deviation in ultrasonic velocity, excess molar volume, deviation isentropic compressibility, excess molar enthalpy and excess acoustic impedance. The results have been fitted to Redlich-Kister polynomial equation. Density and ultrasonic velocity data have been fitted by third order polynomial equation. Similar studies have been done earlier by the workers and have been published in national and international journals(Yasmin and Gupta, 2011, M.Yasmin et al, 2011, S. Singh et. al. 2011, Gurung and Roy, 2006, Ciocirlan and Iuliana, 2009).

\section{Experimental Procedures}

2.1 Materials. The analytical grade chemicals PEGDA (Aldrich), PEGDMA (Aldrich) and pentanol (SigmaAldrich, ACS reagent, $\geq 99 \%$ ) have been obtained from Sigma-Aldrich Chemicals Pvt. Ltd. The average molecular weight $\mathrm{M}_{\mathrm{n}}$ of PEGDA $575 \mathrm{~g} \cdot \mathrm{mol}^{-1}$ and that for PEGDMA is 750 g. $\mathrm{mol}^{-1}$. All chemicals were purified by standard procedure discussed by Perrin and Armarego (D.D. Perrin and W.L.F. Armarego, 1988). Solutions have been prepared by mass, using air tight bottles and have been measured on electronic balance OHAUS- AR 2104 (Ohaus Corp. Pine Brook, NJ, USA) with an accuracy of $1 \times 10^{-4} \mathrm{~g}$. The possible error in the estimation of mole fraction is less than \pm 0.0001 .

2.2 Apparatus and procedures. Densities have been measured by a single capillary calibrated pycnometer, with a bulb capacity of $6.7 \mathrm{ml}$ volume. The pycnometer stem contained graduation of $0.01 \mathrm{ml}$. Pycnometer was immersed vertically in a double walled cylindrical water circulated glass jacket. The liquid rise in the capillary of pycnometer 
was measured by travelling microscope (having a least count of $0.001 \mathrm{~cm}$ ) for accuracy. The precision of the measured densities is of the order of $\pm 1 \times 10^{-4} \mathrm{~g} . \mathrm{cm}^{-3}$.

The interferometric technique has been used for the determination of ultrasonic velocity. The apparatus is a variable path fixed frequency $(2 \mathrm{MHz})$ interferometer (Model F-81, Mittal Enterprises, New Delhi). It consists of a high frequency generator and a double walled measuring cell. A digital micrometer (with a least count of $0.001 \mathrm{~mm}$ ) has been used to measure the distance between the reflector plate and the crystal within the cell. Once the wavelength is known, the ultrasonic velocity $(u)$ in the liquid can be obtained using the following relation:

\section{ultrasonic .velocity $=$ frequency $\times$ wavelength}

The precision in the measurement of ultrasonic velocity is found to be $0.1 \mathrm{~m} . \mathrm{s}^{-1}$.

Circulating water bath with programmable temperature controller (TC-502, Brookfield Engineering Laboratories, Inc., USA), and variable pump speeds, has been used for water circulation around liquid cell of interferometer and water jacket of pycnometer. The programmable controllers incorporate an RS232 interface to provide remote data logging and control capability. The temperature controller covers the measurement range of temperature from $20^{\circ} \mathrm{C}$ to $200^{\circ} \mathrm{C}$, with temperature stability of $\pm 0.01{ }^{\circ} \mathrm{C}$.

\section{Results and Discussion}

3.1 Experimental Results. The experimentally measured values of density and ultrasonic velocity of pure liquids and their mixtures for the systems pentanol + PEGDA and pentanol + PEGDMA are reported in Table 1, as a function of concentration and temperature. In both the mixtures, both the density and ultrasonic velocity decrease non-linearly with increase in mole fraction of pentanol. Pentanol is highly self-associated through hydrogen bonds. Thus their structure and properties are determined mainly by quasi chemical bonds between the molecules, resulting in the formation of multimers of different size and structure. Mixing of polymer in pentanol enhances breaking of these self associated structures of pentanol and induces specific interaction between the components of the mixture.

Jacobson has shown that the intermolecular free length can be estimated from the experimental density and ultrasonic velocity data using the relation,

$$
L_{f}=\frac{K}{u \rho^{1 / 2}}
$$

where $K$ is temperature dependent empirical constant, proposed by Jacobson.

The pseudo-Grüneisen parameter $\Gamma$, has been defined in terms of specific heat ratio as

$$
\Gamma=\frac{\gamma-1}{\alpha T}
$$

where $\gamma$ is specific heat ratio defined as
Table 1. Experimental values of densities $\rho$ and ultrasonic velocities $u$ for the systems pentanol + PEGDA and pentanol + PEGDMA at 293.15, 303.15 and $313.15 \mathrm{~K}$ with

\begin{tabular}{|c|c|c|c|c|c|c|}
\hline \multirow[t]{2}{*}{$x_{1}$} & \multicolumn{2}{|l|}{$\begin{array}{l}\mathrm{T} / \mathrm{K} \\
293.15\end{array}$} & \multicolumn{2}{|l|}{$\begin{array}{l}\mathrm{T} / \mathrm{K} \\
303.15 \\
\end{array}$} & \multicolumn{2}{|l|}{$\begin{array}{l}\mathrm{T} / \mathrm{K} \\
313.15 \\
\end{array}$} \\
\hline & $\begin{array}{l}\rho \\
\text { g.cm }\end{array}$ & $\begin{array}{l}u \\
\mathrm{~m} . \mathrm{s}^{-1}\end{array}$ & $\begin{array}{l}\rho \\
\text { g.cm }\end{array}$ & $\begin{array}{l}u \\
\mathrm{~m} \cdot \mathrm{s}^{-1}\end{array}$ & $\begin{array}{l}\rho \\
\text { g.cm }\end{array}$ & $\begin{array}{l}u \\
\mathrm{~m} \cdot \mathrm{s}^{-1}\end{array}$ \\
\hline \multicolumn{7}{|c|}{ Pentanol + PEGDA } \\
\hline 0.0000 & 1.1206 & 1552.0 & 1.1133 & 1528.0 & 1.1018 & 1492.0 \\
\hline 0.0993 & 1.1151 & 1544.0 & 1.1083 & & 1.0975 & \\
\hline 0.2031 & 1.1070 & 153 & 1.1006 & & 1.0899 & \\
\hline 0.3389 & 1.0930 & & 1.0865 & & 1.0762 & \\
\hline 0.4084 & 1.0840 & & 1.0774 & & 1.0671 & \\
\hline 0.5025 & 1.068 & & 1.0624 & & 1.0524 & \\
\hline 0.5990 & $1.04 \mathrm{C}$ & & 1.04 & & 1.0329 & \\
\hline & 1.02 & & 1.0 & & & \\
\hline 0.79 & 0.981 & & 0.97 & & 0.9670 & \\
\hline 0.89 & 0.9220 & & 0.91 & & 0.9077 & \\
\hline 1.0 & 0.8147 & 286.0 & 0.8078 & & 0.8007 & 1216.0 \\
\hline \multicolumn{7}{|c|}{ Pentanol + PEGDMA } \\
\hline 0.0000 & 1.1037 & 1548.0 & 1.0978 & 1516.0 & 1.0893 & \\
\hline & 1.1010 & & 1.0956 & & 1.0880 & \\
\hline & 1.0956 & & 1.0904 & & 1.0829 & \\
\hline 0.3139 & 1.0881 & & 1.0832 & & 1.0756 & 1469.1 \\
\hline 0.4000 & 1.0800 & & 1.0757 & & 1.0682 & 1455.8 \\
\hline 0.5010 & 1.0678 & & 1.0638 & & 1.0566 & 1436.1 \\
\hline 0.6002 & 1.0522 & & 1.0483 & & 1.0409 & 1408.0 \\
\hline 0.6971 & 1.0300 & & 1.0261 & & 1.0188 & 1377.2 \\
\hline 0.7990 & 0.9949 & & 0.9911 & & 0.9835 & \\
\hline 0.9003 & 0.9351 & 1355 & 0.9303 & 132 & 0.9226 & 1296.0 \\
\hline 1.0000 & 0.8147 & 1286.0 & 0.8078 & 1254.4 & 0.8007 & 1216.0 \\
\hline
\end{tabular}
respect to the mole fraction $x_{1}$ of pentanol

$\gamma=\frac{\beta_{T}}{\beta_{S}}$

The relation utilized to estimate the internal pressure in pure liquids and liquid mixtures is

$\pi_{i}=\frac{\alpha T}{k_{T}}$

The parameters intermolecular free length $\left(L_{f}\right)$, internal pressure $\left(\pi_{i}\right)$ and pseudo-Grüneisen parameter $(\Gamma)$ are temperature sensitive and provides significant information regarding intermolecular interaction between the component molecules in the mixtures. These parameters have been evaluated over the entire composition range for both the mixtures and have been represented in Figures 1 to 3 .

The intermolecular free length $\left(L_{f}\right)$, of the mixtures increases with increasing concentration of pentanol (figure 1). Breaking of self associated structures of pentanol occurs due to dissociation of hydrogen bonds in pentanol on mixing the components. The rate of increase of intermolecular free length also increases with increase concentration of pentanol. This may be due to the fact that pentanol is highly self associated by hydrogen bonding to the extent of polymerization. Hence the larger voids are formed by self-associated pentanol molecules than by PEGDA or PEGDMA molecules. PEGDMA molecules are larger than that of PEGDA hence intermolecular free length is larger in the system pentanol + PEGDMA than in pentanol + PEGDA. Rise in temperature makes the free length increase as expected due to thermal expansion of liquids. 


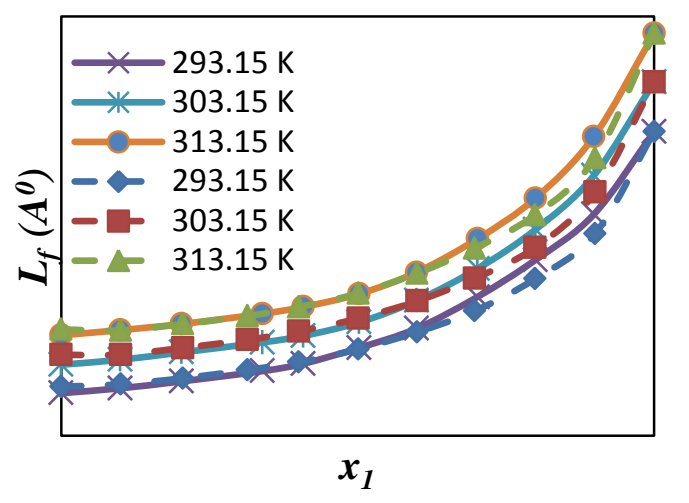

Figure 1. Intermolecular free length for the system pentanol + PEGDA (-) and pentanol + PEGDMA (---) at 293.15, 303.15 and $313.15 \mathrm{~K}$ with respect to the mole fraction of pentanol.

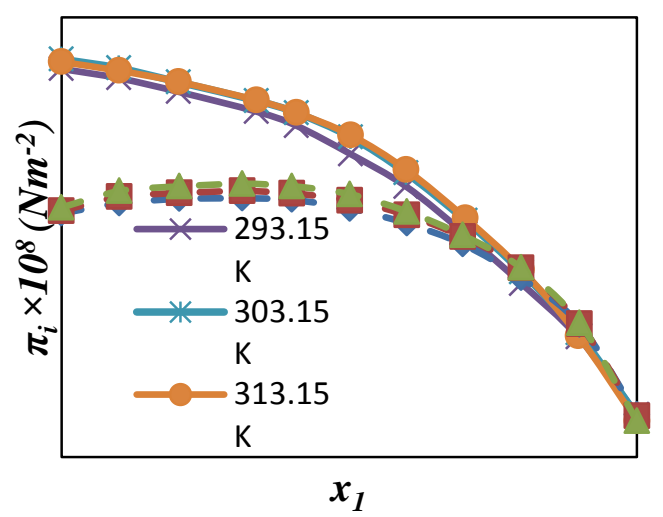

Figure 2. Internal pressure for the system pentanol + PEGDA (-) and pentanol + PEGDMA(---) at 293.15, 303.15 and $313.15 \mathrm{~K}$ with respect to the mole fraction of pentanol.
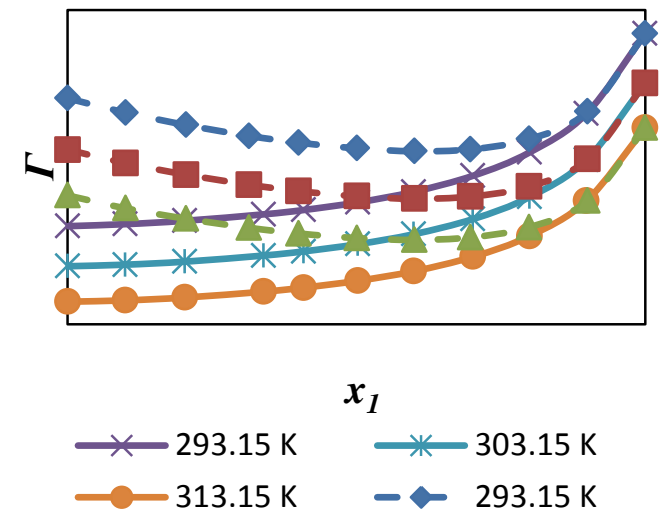

Figure 3. Pseudo-Grüneisen parameter for the system pentanol + PEGDA (-) and pentanol + PEGDMA (---) at $293.15,303.15$ and $313.15 \mathrm{~K}$ with respect to the mole fraction of pentanol.

The role of internal pressure $\left(\pi_{i}\right)$ in solution thermodynamics was recognized many years ago by Hilderband following earlier work of Van Laar (J.M. Prausnitz, 1969). It has been found to be an important tool in the study of several chemical reactions and in the investigation of molecular interactions. The internal pressure of the mixture is found to vary non-linearly with concentration of pentanol as listed in Figure 2. The internal pressure of the mixture decreases with increase in mole fraction of pentanol in the mixture. The internal pressure values increase slightly with rise in temperature as expected.

Pseudo-Grüneisen parameter $(\Gamma)$ is one of the important parameter which is used to study internal structure, clustering phenomenon and thermodynamic properties of solid crystalline lattice. It is well established that liquids support a quasi-crystalline model for their structure, the lattice nature being increased at high pressure and low temperature hence the pseudo-Grüneisen parameter can also be used to study liquids. Figure 3 reveals that the pseudoGrüneisen parameter of mixture decreases with increase in mole fraction of pentanol as well as with rise in temperature. The non-linear behaviour of this parameter suggests the presence of specific interaction in the mixtures. Similar variations in pseudo-Grüneisen parameter for the system $\mathrm{THF}+\mathrm{o}$-cresol have been also found (M. Yasmin et al., 2009).

The absolute rate theory based Eyring's kinematic viscosity model gives the relation

$\eta V=N_{A} h \exp \left(\frac{\Delta G^{*}}{R T}\right)$

where $\eta, V, N_{A}, h, R, T, \Delta G^{*}$ are kinematic viscosity, molar volume, Avogadro's constant, Planck's constant, Universal gas constant, absolute temperature, and free energy of activation.

The entropy of viscous flow $\Delta S^{*}$, enthalpy $\Delta H^{*}$ and Gibbs free energy of activation for viscous flow $\Delta G^{*}$ are related by the following equation

$\Delta G^{*}=\Delta H^{*}-T \Delta S^{*}$

The values of enthalpy, entropy and Gibbs free energy of activation for viscous flow at different concentrations of polymer solution have been given in Table 2 .

Entropy of activation for viscous flow $\Delta S^{*}$ for both the systems is found to be negative. Enthalpy of activation of viscous flow $\Delta H^{*}$ on the other side is positive for all the three systems under study and values are about constant over the entire range of studied temperature for each concentration.

The values of $\Delta H^{*}$ are positive and of $\Delta S^{*}$ negative, indicating that the attainment of the transition state for viscous flow is accompanied by bond breaking. The entropy change of activation from the initial state to the transition at a given composition is significant during an activated viscous flow process; therefore this process is entropycontrolled for PEG solutions. It seems that for the viscous flow process, the structural factor dominates over the interactional one, as in the case of the mixing properties.

Gibbs free energy of activation for viscous flow $\Delta G^{*}$ increases with increase in temperature for the systems pentanol + PEGDMA and pentanol + PEGDA.

3.2 Excess parameters. The study on the possible change of thermodynamic properties of mixtures and their degree of deviation from ideality is an excellent qualitative and quantitative way to investigate about the molecular structure 
Table 2. Calculated values of entropy of viscous flow, enthalpy and Gibbs free energy of activation for viscous flow at different concentrations of polymer solution for the systems pentanol + PEGDA and pentanol + PEGDMA at 293.15, 303.15 and $313.15 \mathrm{~K}$.

\begin{tabular}{|c|c|c|c|c|c|}
\hline$x_{I}$ & $\begin{array}{l}\Delta S^{*} \\
\mathrm{kJmol}^{-1}\end{array}$ & $\begin{array}{l}\Delta H^{*} \\
\mathrm{kJmol}^{-1}\end{array}$ & $\begin{array}{l}\Delta G^{*} \\
\text { kJmol- }^{1} \\
293.15 \mathrm{~K}\end{array}$ & $\begin{array}{l}\Delta G^{*} \\
\mathrm{kJmol}^{-1} \\
303.15 \mathrm{~K}\end{array}$ & $\begin{array}{l}\Delta G^{*} \\
\mathrm{kJmol}^{-1} \\
313.15 \mathrm{~K}\end{array}$ \\
\hline \multicolumn{6}{|c|}{ pentanol+PEGDA } \\
\hline 0.0000 & -33.73 & 34.866 & 44.798 & 45.071 & 45.453 \\
\hline 0.0993 & -39.47 & 32.644 & 44.268 & 44.561 & 45.040 \\
\hline 0.2031 & -40.31 & 31.800 & 43.664 & 43.982 & 44.451 \\
\hline 0.3390 & -43.24 & 30.040 & 42.762 & 43.119 & 43.608 \\
\hline 0.4084 & -42.97 & 29.596 & 42.229 & 42.591 & 43.075 \\
\hline 0.5025 & -40.53 & 29.554 & 41.464 & 41.815 & 42.266 \\
\hline 0.5991 & -46.30 & 26.878 & 40.493 & 40.885 & 41.403 \\
\hline 0.7019 & -47.06 & 25.438 & 39.270 & 39.656 & 40.204 \\
\hline 0.7995 & -52.97 & 22.313 & 37.884 & 38.332 & 38.928 \\
\hline 0.8981 & -52.70 & 20.551 & 36.008 & 36.504 & 37.064 \\
\hline 1.0000 & -50.04 & 19.152 & 33.842 & 34.357 & 34.820 \\
\hline \multicolumn{6}{|c|}{ pentanol+PEGDMA } \\
\hline 0.0000 & -43.32 & 33.327 & 46.065 & 46.388 & 46.930 \\
\hline 0.1005 & -46.69 & 31.881 & 45.602 & 45.978 & 46.535 \\
\hline 0.2051 & -50.72 & 30.158 & 45.076 & 45.459 & 46.085 \\
\hline 0.3139 & -50.81 & 29.476 & 44.426 & 44.804 & 45.436 \\
\hline 0.4001 & -49.60 & 29.240 & 43.823 & 44.208 & 44.810 \\
\hline 0.5011 & -49.23 & 25.833 & 43.015 & 43.407 & 43.984 \\
\hline 0.6003 & -51.51 & 27.157 & 42.003 & 42.395 & 43.013 \\
\hline 0.6971 & -53.57 & 25.056 & 40.837 & 41.170 & 41.900 \\
\hline 0.7991 & -53.77 & 23.364 & 39.242 & 39.502 & 40.295 \\
\hline 0.9003 & -53.97 & 21.164 & 37.027 & 37.427 & 38.113 \\
\hline 1.0000 & -50.04 & 19.152 & 33.842 & 34.357 & 34.820 \\
\hline
\end{tabular}

and intermolecular forces in liquid mixtures. This has given impetus to the theoretical and experimental evaluation ( $\mathrm{S}$. Parveen et al., 2010) of the excess thermodynamic properties of liquid mixtures. Excess properties are sensitive towards external factors such as composition and temperature.

The excess parameters have been evaluated using following relations,

Deviation in ultrasonic velocity,

$\Delta u=u-\left(x_{1} u_{1}+x_{2} u_{2}\right)$

Excess molar volume,

$V^{E}=\left(\frac{x_{1} m_{1}+x_{2} m_{2}}{\rho}\right)-\left(\frac{x_{1} m_{1}}{\rho_{1}}+\frac{x_{2} m_{2}}{\rho_{2}}\right)$

Excess acoustic impedance,

$Z^{E}=(\rho u)-\left(x_{1} \rho_{1} u_{1}+x_{2} \rho_{2} u_{2}\right)$

Excess isentropic compressibility,

$$
\Delta k_{s}=\frac{1}{u^{2} \rho}-\left(\frac{x_{1}}{u_{1}^{2} \rho_{1}}+\frac{x_{2}}{u_{2}^{2} \rho_{2}}\right)
$$

Excess molar enthalpy,

$H_{m}^{E}=x_{1} \pi_{i 1} V_{1}+x_{2} \pi_{i 2} V_{2}-\pi_{i} V$
Excess molar volume, deviation in isentropic compressibility, deviation in ultrasonic velocity, excess acoustic impedance and excess molar enthalpy for the binary mixtures under study have been reported in Figures 4-8.

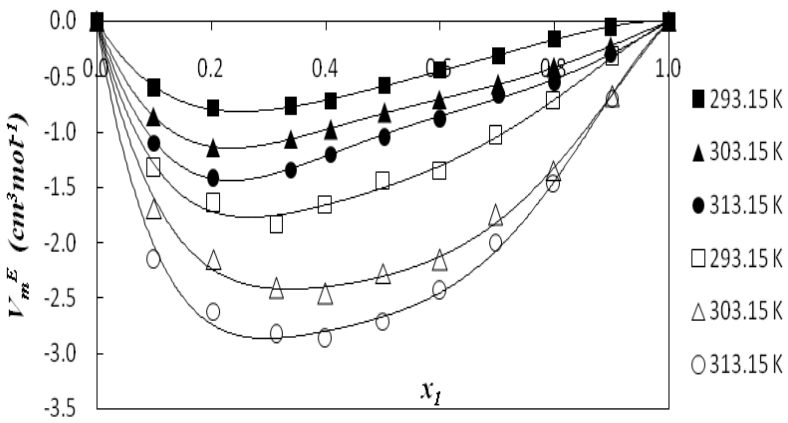

Figure 4. Excess molar volume for the system (ロ, $\mathbf{\Delta}, \bullet)$ pentanol + PEGDA and $(\square, \Delta, O)$ pentanol + PEGDMA at $293.15,303.15$ and $313.15 \mathrm{~K}$ with respect to the mole fraction of pentanol.

The excess molar volume $V^{E}$ (Figure 4) for the systems pentanol+ PEGDA and pentanol+ PEGDMA are negative over entire composition range at all three temperatures. The large negative values arise due to increased interactions between PEGDMA or PEGDA and solvent or a very large difference in the molar volumes of the components. Treszczanowicz et al (A.J. Treszczanowicz et al., 1981) suggested that $V^{E}$ is the result of contributions from several opposing effects, namely, physical, chemical, and structural. Physical contributions, which are non specific interactions between the real species present in the mixture, contribute a positive term to $V^{E}$. The chemical or specific intermolecular interactions result in a volume decrease. This effect contributes negative values to $V^{E}$. The structural contributions are mostly negative and arise from several effects, especially from interstitial accommodation and changes in the free volume. In other words, structural contributions arising from the geometrical fitting (interstitial accommodation) of one component into other due to the differences in the free volumes and molar volumes between components lead to negative contribution to $V^{E}$. The actual value of $V^{E}$ would depend on the relative strengths of these effects. The experimental values of $V^{E}$ suggest that interstitial accommodation is leading to the negative values while increase in negative values with temperature suggest that structural effect is more prominent than chemical effect in these solutions because of the large difference in molar volumes between components. The larger difference in molar volume of components in the system pentanol+ PEGDMA than that in the system pentanol + PEGDA (108.18, 513.09 and $679.48 \mathrm{~cm}^{3} \mathrm{~mol}^{-1}$ for pentanol, PEGDA and PEGDMA respectively at 293.15 $\mathrm{K})$ results in larger excess molar volumes. Earlier workers have also concluded the above result for polymer solutions (M.T. Zafarani-Moattar and N. Tohidifar, 2008). The increase in negative values of $V^{E}$ with temperature reflects that thermal energy enhances the strength of interaction.

Deviations in isentropic compressibility $\Delta k_{s}$, Figure 5 , is to be negative over whole composition range. Thus it can be concluded that mixing of pentanol with PEGDA and 
PEGDMA respectively results in enhanced rigidity. In liquid phase a molecule of pentanol is able to build up at most two hydrogen bonds with its next neighbours to form open cyclic polymeric chains of various lengths. Mixing of polymers causes more compact structure due to interstitial accommodation of component molecules. Fort and Moore (R.J. Fort and W.R. Moore 1955). has shown earlier that liquids of different molecular size usually mix with decrease in volume resulting in negative $\Delta k_{s}$ values. Similar results have been found by Singh et al (K. P. Singh et al., 2009) for the binary mixtures of 2-butoxyethanol with PEG 200 and PEG 400.

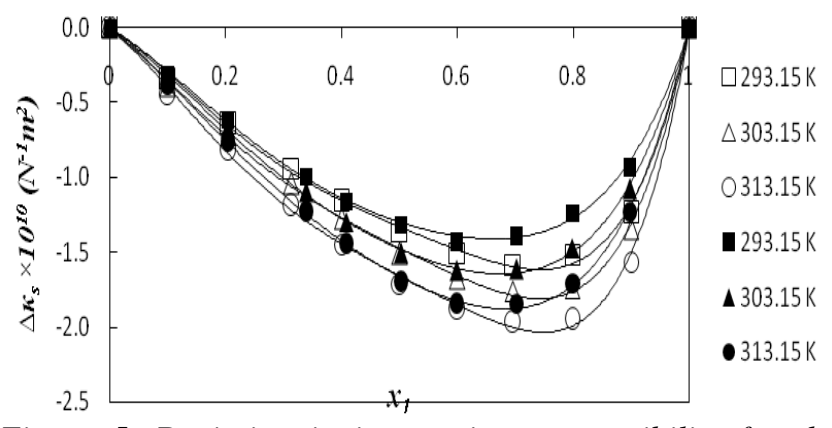

Figure 5. Deviation in isentropic compressibility for the system (ロ, $\mathbf{\Lambda}, \bullet)$ pentanol + PEGDA and $(\square, \Delta, O)$ pentanol + PEGDMA at 293.15, 303.15 and $313.15 \mathrm{~K}$ with respect to the mole fraction of pentanol.

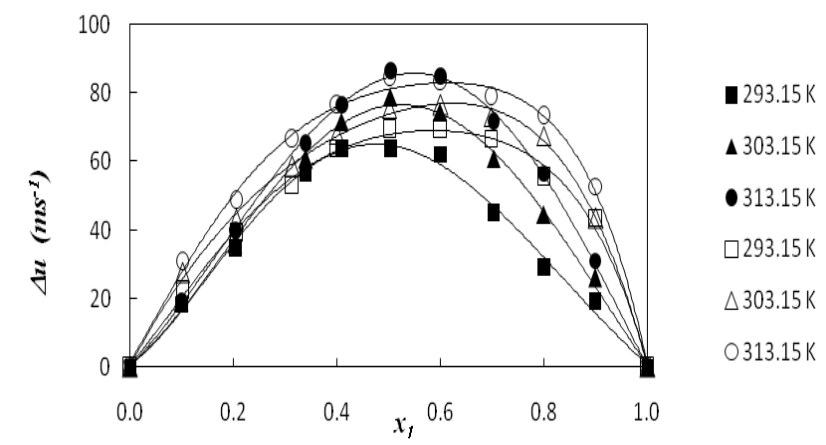

Figure 6. Deviation in ultrasonic velocity for the system $(\boldsymbol{\bullet}, \mathbf{\Delta}, \bullet)$ pentanol + PEGDA and $(\square, \Delta, O)$ pentanol + PEGDMA at 293.15, 303.15 and $313.15 \mathrm{~K}$ with respect to the mole fraction of pentanol.

Due to intermolecular interactions structure of the molecule is changed, which affect the compressibility and thus a change in ultrasonic velocity. The ultrasonic velocity in a mixture is mainly influenced by the free length between the surfaces of the molecules of the mixture. The inverse dependence of intermolecular free length and ultrasonic velocity has been evolved from the model of sound propagation proposed by Eyring and Kincaid (J.F. Kincaid and H. Eyring, 1937). As shown in Figure 6, deviation in ultrasonic velocity $\Delta u$ is positive for the mixtures. Our results for intermolecular free length and deviation in ultrasonic velocity support this theory. This kind of variations suggests that significant interactions are operative in these mixtures as also studied by Ali and Tariq (A. Ali and M. Tariq, 2006) for the system benzyl alcohol with benzene. The increase in $\Delta u$ with temperature suggests that thermal energy enhances the strength of interaction.

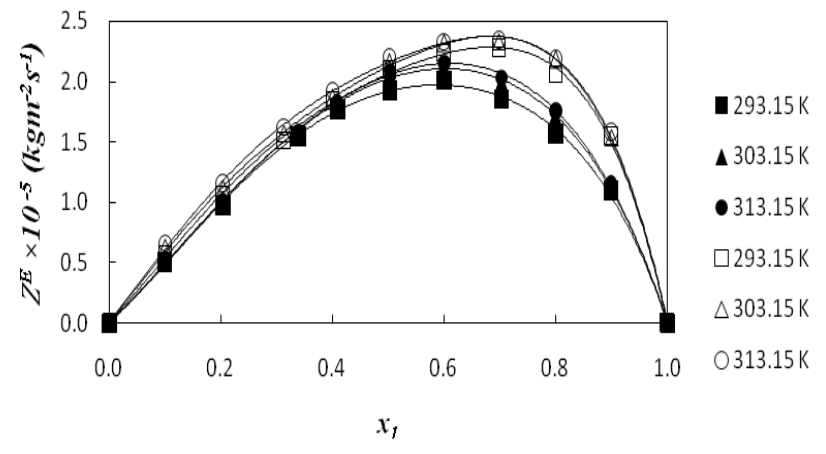

Figure 7. Excess acoustic impedance for the system $(\boldsymbol{\bullet}, \mathbf{\Delta}, \bullet)$ pentanol + PEGDA and $(\square, \Delta, O)$ pentanol + PEGDMA at 293.15, 303.15 and $313.15 \mathrm{~K}$ with respect to the mole fraction of pentanol.

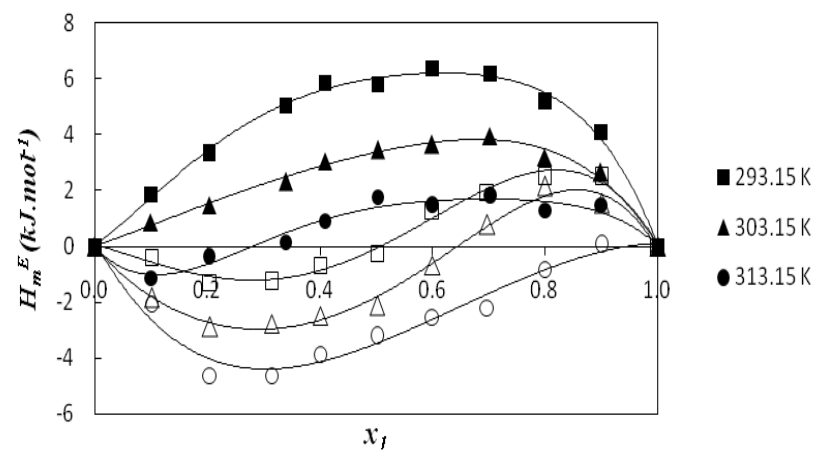

Figure 8. Excess molar enthalpy for the system $(\mathbf{\square}, \mathbf{\Delta}, \bullet)$ pentanol + PEGDA and $(\square, \triangle, O)$ pentanol + PEGDMA at $293.15,303.15$ and $313.15 \mathrm{~K}$ with respect to the mole fraction of pentanol.

Specific acoustic impedance is a quantity, which depends on the molecular packing of the systems. It can be seen from Figure 7 that the values of excess acoustic impedance $Z^{E}$ are positive for both the mixtures which indicate the presence of specific intermolecular interaction through hydrogen bonding between the unlike species. The effect of temperature on $Z^{E}$ is not very prominent in all the mixtures. The values are more positive for the system pentanol+ PEGDMA as compared to pentanol+ PEGDA suggesting the interaction to be stronger in solution of PEGDMA than in that of PEGDA.

The excess molar enthalpy $H_{m}{ }^{E}$ curve (Figure 8) has been found to be $S$ - shaped for pentanol+ PEGDMA mixture and positive for pentanol+ PEGDA mixture. The values of $H_{m}{ }^{E}$ for pentanol + PEGDMA are negative in polymer rich region and turn to be small positive in pentanol rich region. The negative values of $H_{m}{ }^{E}$ in the case of pentanol + PEGDMA mixture indicate the presence of strong intermolecular interaction between unlike molecules. However, positive values of $H_{m}{ }^{E}$ suggest that the effect of rupture of hydrogen bonded chain of pentanol dominates over the specific interactions between pentanol and PEGDMA molecules. This may also be due to greater steric hindrance caused by methyl groups attached in the PEGDMA molecules. The negative values of $H_{m}{ }^{E}$ decreases as temperature increases in the case of pentanol + PEGDMA mixture. However, positive $H_{m}{ }^{E}$ values in the case of pentanol+ PEGDA mixture decreases as temperature increases which suggests decrease in self association of the pure component and increase in strength 
of interaction with temperature. Increase in temperature will cause rupture of more and more H-bonded self association in pentanol molecules and also hetero association between unlike molecules. This reduces the positive deviation with increase in temperature.

The observed variation in these thermodynamic parameters in the present investigation may be result of contribution from several effects such as:

1. The breakdown of self associated structures of pentanol.

2. The specific interactions in the systems pentanol+ PEGDMA and pentanol+ PEGDA

3. Structural contribution arising from the geometrical fitting of molecules of one component into the voids forms by the molecules of other.

3.3 Redlich-Kister polynomial equation. The composition dependences of the excess properties are correlated by the Redlich-Kister polynomial equation (O. Redlich and A.T. Kister, 1984)

$$
Y^{E}=x_{1}\left(1-x_{1}\right) \sum_{i=1}^{5} a_{i}\left(2 x_{1}-1\right)^{i-1}
$$

The values of the coefficient $a_{i}$ were calculated by method of least squares along with the standard deviation $\sigma\left(Y^{E}\right)$. The coefficient $a_{i}$ is adjustable parameters for a better fit of the excess functions.

The standard deviation values were obtained from relation
$\sigma\left(Y^{E}\right)=\left[\frac{\sum_{i=1}^{n}\left(Y_{\exp t}^{E}-Y_{c a l}^{E}\right)^{2}}{n-p}\right]^{1 / 2}$

where $n$ is the no. of experimental points, $p$ is the no. of parameters, $Y_{\text {expt }}$ and $Y_{\text {cal }}$ are the experimental and calculated parameters respectively. The values of coefficient $a_{i}$ evaluated using the method of least squares for the mixtures are given in Table 3 along with the standard deviations $\sigma\left(Y^{E}\right)$.

3.4 Data Correlation. The density and ultrasonic velocity data are fitted by the following relations

$$
\begin{aligned}
& \rho / g . c m^{3}=a x_{1}^{3}+b x_{1}^{2}+c x_{1}+d \\
& u / m s^{-1}=a^{\prime} x_{1}^{3}+b^{\prime} x_{1}^{2}+c^{\prime} x_{1}+d^{\prime}
\end{aligned}
$$

where $\rho$ and $u$ are the density and ultrasonic velocity of the solution at the measured temperature, $a, b, c$ and $d$ are the coefficients of the polynomial in $\mathrm{gcm}^{-3} a^{\prime}, b^{\prime}, c^{\prime}$ and $d^{\prime}$ are the coefficients of the polynomial in $\mathrm{ms}^{-1}$ and $x_{I}$ is the mole fraction of the polymer in the solution.

Values of the coefficients are obtained by regression. The coefficients in the equation 14 and 15 along with the percentage average absolute deviations (\%AAD) calculated using equation 16 are given in Tables 4 and 5 .

\begin{tabular}{|c|c|c|c|c|c|c|c|}
\hline Parameters & $T / \mathrm{K}$ & $a_{1}$ & $a_{2}$ & $a_{3}$ & $a_{4}$ & $a_{5}$ & $\sigma\left(Y^{E}\right)$ \\
\hline \multicolumn{8}{|c|}{ pentanol+PEGDA } \\
\hline \multirow{3}{*}{$V_{m}{ }^{E} \times 10^{-6} /\left(\mathrm{m}^{3} \cdot \mathrm{mol}^{-1}\right)$} & 293.15 & -2.3419 & -2.3676 & -1.1799 & -2.3029 & -1.2443 & 0.0097 \\
\hline & 303.15 & -4.1909 & -7.5774 & -8.9660 & 5.2195 & 10.1736 & 0.3269 \\
\hline & 313.15 & -4.0957 & -3.5170 & -6.7300 & -3.2042 & 1.7138 & 0.0323 \\
\hline \multirow[t]{3}{*}{$\Delta k_{s} \times 10^{-10} /\left(\mathrm{N}^{-1} \mathrm{~m}^{2}\right)$} & 293.15 & -5.1555 & 2.0441 & -0.2689 & 3.4236 & -3.7952 & 0.0089 \\
\hline & 303.15 & -6.0359 & 3.5958 & 0.2715 & 2.2501 & -4.7917 & 0.0050 \\
\hline & 313.15 & -6.7231 & 4.3271 & -1.2736 & 2.3478 & -3.0795 & 0.0106 \\
\hline \multirow[t]{3}{*}{$\Delta u /\left(\mathrm{m} \cdot \mathrm{s}^{-1}\right)$} & 293.15 & 265.9901 & 69.8559 & -335.9051 & -115.4274 & 388.6938 & 1.5115 \\
\hline & 303.15 & 316.4021 & -62.9194 & -293.2690 & 27.2195 & 292.8781 & 0.5887 \\
\hline & 313.15 & 339.2848 & -104.3641 & -100.7280 & 40.1547 & 0.9336 & 1.2774 \\
\hline \multirow[t]{3}{*}{$\mathrm{Z}^{\mathrm{E}} \times 10^{-3} /\left(\mathrm{kg} \cdot \mathrm{m}^{-2} \cdot \mathrm{s}^{-1}\right)$} & 293.15 & 7.7240 & -1.9118 & -1.1733 & -3.3757 & 4.6233 & 0.0157 \\
\hline & 303.15 & 8.2235 & -3.3410 & -1.3311 & -1.4415 & 4.4314 & 0.0073 \\
\hline & 313.15 & 8.2923 & -3.3860 & 0.6839 & -1.5270 & 1.0790 & 0.0154 \\
\hline \multirow{3}{*}{$H_{m}{ }^{E} /\left(\mathrm{kJ} \cdot \mathrm{mol}^{-1}\right)$} & 293.15 & 23.4799 & -4.0431 & -4.2105 & -17.0379 & 30.7781 & 0.2177 \\
\hline & 303.15 & 13.3327 & -32.4870 & -50.3619 & 31.7156 & 94.0715 & 0.1611 \\
\hline & 313.15 & 10.2706 & -49.2561 & 59.4002 & 49.6605 & -114.5839 & 0.2777 \\
\hline & & & pentanol+PEC & MA & & & \\
\hline \multirow[t]{3}{*}{$V_{m}{ }^{E} \times 10^{-6} /\left(\mathrm{m}^{3} \cdot \mathrm{mol}^{-1}\right)$} & 293.15 & -5.8832 & -3.9235 & -5.7787 & -4.5277 & 1.2148 & 0.0696 \\
\hline & 303.15 & -10.6823 & -0.7168 & 3.7517 & -9.7382 & -11.8859 & 0.0444 \\
\hline & 313.15 & -10.8614 & -3.7187 & -6.1229 & -9.9667 & -2.7296 & 0.0182 \\
\hline \multirow[t]{3}{*}{$\Delta k_{s} \times 10^{-10} /\left(\mathrm{N}^{-1} \cdot \mathrm{m}^{2}\right)$} & 293.15 & -5.4554 & 2.4907 & -1.3545 & 5.9445 & -5.9520 & 0.0053 \\
\hline & 303.15 & -6.0177 & 3.7438 & -2.8602 & 4.5498 & -4.4420 & 0.0087 \\
\hline & 313.15 & -6.9236 & 3.8599 & -1.5874 & 6.1359 & -7.9408 & 0.0103 \\
\hline \multirow[t]{3}{*}{$\Delta u /\left(\mathrm{m} \cdot \mathrm{s}^{-1}\right)$} & 293.15 & 277.7313 & -16.9259 & -76.5531 & -205.5004 & 334.1284 & 0.9966 \\
\hline & 303.15 & 324.4851 & -420.2246 & 314.2025 & 940.9104 & -975.4218 & 11.6873 \\
\hline & 313.15 & 373.3022 & 228.0240 & 401.0586 & -601.4012 & -407.0663 & 0.3807 \\
\hline \multirow[t]{3}{*}{$\mathrm{Z}^{\mathrm{E}} \times 10^{-3} /\left(\mathrm{kg} \cdot \mathrm{m}^{-2} \cdot \mathrm{s}^{-1}\right)$} & 293.15 & 8.3777 & -3.4936 & 1.6726 & -5.0429 & 5.6705 & 0.0087 \\
\hline & 303.15 & 8.5904 & -4.5825 & 3.5882 & -2.2668 & 2.8949 & 0.0099 \\
\hline & 313.15 & 8.7533 & -3.9797 & 2.9046 & -3.6790 & 4.5697 & 0.0121 \\
\hline \multirow[t]{3}{*}{$H_{m}{ }^{E} /\left(\mathrm{kJ} \cdot \mathrm{mol}^{-1}\right)$} & 293.15 & 1.3471 & -19.8274 & -5.1583 & -0.5210 & 32.8853 & 0.2234 \\
\hline & 303.15 & -0.3106 & 37.7858 & 106.7547 & -96.5269 & -171.4546 & 0.2367 \\
\hline & 313.15 & -6.8308 & -25.2372 & -54.6883 & 16.6202 & 75.1354 & 0.0954 \\
\hline
\end{tabular}

Table 3. Coefficients of the Redlich-Kister equation for excess parameters and their standard deviation for the system pentanol + PEGDA and pentanol + PEGDMA 
Table 4. Coefficients of polynomial equation (14) for the systems pentanol + PEGDA and pentanol + PEGDMA at 293.15, 303.15 and $313.15 \mathrm{~K}$.

\begin{tabular}{ccccccc}
\hline$T / \mathrm{K}$ & $a$ & $b$ & $c$ & $d$ & \%AAD & Max\%D \\
\hline \multicolumn{7}{c}{ Pentanol + PEGDA } \\
\hline 293.15 & -0.588 & 0.481 & -0.196 & 1.125 & -0.0811 & 0.8854 \\
303.15 & -0.591 & 0.483 & -0.194 & 1.118 & -0.0375 & 1.0136 \\
313.15 & -0.582 & 0.472 & -0.188 & 1.107 & -0.0049 & 1.0353 \\
\hline \multicolumn{7}{c}{ Pentanol + PEGDMA } \\
\hline 293.15 & -0.716 & 0.646 & -0.214 & 1.110 & -0.0678 & 1.3763 \\
303.15 & -0.734 & 0.664 & -0.215 & 1.105 & -0.0004 & 1.5087 \\
313.15 & -0.727 & 0.652 & -0.208 & 1.096 & -0.0195 & 1.5349 \\
\hline
\end{tabular}

Table 5. Coefficients of polynomial equation (15) for the systems pentanol + PEGDA and pentanol + PEGDMA at $293.15,303.15$ and $313.15 \mathrm{~K}$

\begin{tabular}{rrrrrrc}
\hline$T / \mathrm{K}$ & \multicolumn{1}{c}{$a^{\prime}$} & \multicolumn{1}{c}{$b^{\prime}$} & \multicolumn{1}{c}{$c^{\prime}$} & $d^{\prime}$ & \%AAD & Max \\
\hline \multicolumn{7}{c}{ Pentanol + PEGDA } \\
\hline 293.15 & 59.42 & -345.80 & 21.88 & 1548 & -0.0096 & 0.4600 \\
303.15 & -78.88 & -185.70 & -8.64 & 1524 & -0.0423 & 0.4528 \\
313.15 & -165.90 & -89.02 & -21.26 & 1489 & -0.0137 & 0.3624 \\
\hline \multicolumn{7}{c}{ Pentanol + PEGDMA } \\
\hline 293.15 & -178.60 & 0.35 & -80.95 & 1550 & -0.0038 & 0.6386 \\
303.15 & -222.90 & 40.95 & -79.04 & 1519 & -0.0609 & 0.0458 \\
313.15 & -216.70 & 3.46 & -56.01 & 1492 & -0.0095 & 0.7545 \\
\hline
\end{tabular}

$$
\% A A D=\frac{100}{n} \sum_{i=1}^{n}\left|\frac{A_{c a l}-A_{\text {exp }}}{A_{\exp }}\right|
$$

where $A_{\text {cal }}$ and $A_{\text {exp }}$ is calculated and experimental density and velocity respectively.

\section{Conclusions}

It can be concluded from the above results that there is a presence of specific intermolecular interaction in both the systems. The interaction is found to be stronger in the system pentanol + PEGDMA than in pentanol + PEGDA. The difference in molar volumes of the components is much large hence the structural effect is most prominent in these mixture properties.

The correlation data reported in the paper can be used further in industry or research for theoretical modeling and for studying the mixture further at concentrations other than those reported.

\section{References}

Ali, A., Tariq, M. (2006). Thermodynamic and Transport Behaviour of Binary Liquid Mixtures of Benzyl Alcohol with Monocyclic Aromatics at 303.15 K. J. Mol. Liqs. $128,50-55$.

Ciocirlan, O., Iuliana, O. (2009). Density, viscosity and refractive index of the dimethyl sulfoxide $+o$-xylene system . J. Serb. Chem. Soc. 74, 317-329.

Dumanian, G.A., Dascombe, W., Hong, C., Labadie, K., Garret, K., Sawhney, A.S., Pathak, C.P., Hubbell, J.A., Johnson, P.C. (1995). A New Photopolymerisable Blood Vessel Glue That Seals Human Vessel Anastomoses without Augmenting Thrombogenicity. Plastic and
Reconstructive surgery, Plastic and Reconstructive surgery, 95, 901-907.

Fort, R.J., Moore, W.R. (1955). Adiabatic Compressibilities of Binary Liquid. Mixtures.Trans.Faraday Soc. 61, 2102-2111.

Gurung, B.B., Roy, M.N. (2006). Study of Densities, Viscosities and Ultrasonic Speeds of Binary Mixtures Containing 1,2-Dimethoxyethane and an Alkan-l-ol at 298.15 K. J Solution Chem 35, 1587-1606.

Kincaid, J.F., Eyring, H. (1937) J.Phys Chem, 41, 249.

Parveen, S., Yasmin, M., Gupta, M., Shukla, J.P. (2010). Thermoacoustical and Excess Properties of Binary Mixtures of Ethyl Butyrate with Methanol and Vinyl Acetate. Int. J. of Thermodynamics. 13, 59-66.

Perrin, D.D., Armarego, W.L.F. (1988). Purification of Laboratory Chemicals. (3rd ed.). Oxford: Pergamon Press.

Prausnitz, J.M. (1969). Molecular Thermodynamics of Fluid Phase Equilibria. (2 ${ }^{\text {nd }}$ ed.). New Jersy: Prentice Hall Engle Wood Cliffs.

Redlich, O., Kister ,A.T. (1984). Thermodynamics of Nonelectrolytic Solutions. Algebraic Representation of Thermodynamic Properties and the Classification of Solutions. Ind. Eng. Chem., 40, 345-348.

Singh, K. P., Agarwal, H., Shukla, V.K., Yasmin, M., Gupta, M., Shukla, J.P. (2009). Thermo-acoustical Properties of Peg with Alkoxy Ethanols. J. Pure Appl. Ultrason. 31, 124-130.

Singh, S., Parveen, S., Shukla, D., Yasmin, M., Gupta, M., Shukla, J.P. (2011). Ultrasonic and Volumetric Study of N-H Bond Complexes. J. Solution Chem. 40 889-899.

Treszczanowicz, A.J., Kiyohara, O., Benson, G.C. (1981). Excess Molar Volumes for n-Alkanes Binary Mixture of Decan-1-ol + n-Pentane, + n-Hexane, + n-Octane, + nDecane and + n-Hexadecane. J. Chem. Thermodyn., 13 253-260.

Yasmin, M.,Gupta, M. (2011). Thermoacoustical Analysis of Solutions of Poly (Ethylene Glycol) 200 through HBond Complex Formation. Thermochimica Acta, 518 89-100.

Yasmin, M., Gupta, M., Shukla, J. P. (2011). Experimental and Computational Study on Viscosity and Optical Dielectric Constant of Solutions of Poly (ethylene glycol) 200. J. Mol. Liq, 160, 22-29.

Yasmin, M., Singh, K.P., Parveen, S., Gupta, M., Shukla, J.P. (2009). Excess Properties of Binary Liquid Mixtures -A Comparative Experimental and Theoretical Study. Acta Physica Polonica A, 115, 890-900.

Zafarani-Moattar, M.T., Tohidifar, N. (2008). VapourLiquid Equilibria, Density, Speed of Sound, and Viscosity for the System Poly (ethylene glycol) $400+$ Ethanol at Different Temperatures. J. Chem. Eng. Data. 53, 785-793. 